\title{
THE STOKES EQUATIONS WITH FOURIER BOUNDARY CONDITIONS ON A WALL WITH ASPERITIES
}

\author{
YOUCEF AMIRAT, BLANCA CLIMENT, \\ ENRIQUE FERNÁNDEZ-CARA ${ }^{\dagger}$ AND JACQUES SIMON*
}

\begin{abstract}
We study the effect of the rugosity of a wall on the solution of the Stokes system complemented with Fourier boundary conditions. We consider the case of small periodic asperities of size $\varepsilon$. We prove that the velocity field, pressure and drag respectively converge to the velocity field, pressure and drag of a homogenized Stokes problem, where a different friction coefficient appears. This shows that, contrarily to the case of Dirichlet boundary conditions, rugosity is dominant here.
\end{abstract}

${ }^{*}$ Laboratoire de Mathématiques Appliquées, Université Blaise Pascal (Clermont-Ferrand 2), 63177 Aubière Cedex, France, E-mails: amirat@ucfma.univ-bpclermont.fr, simon@ucfma.univbpclermont.fr.

${ }^{\dagger}$ Dpto. de Ecuaciones Diferenciales y Análisis Numérico, Universidad de Sevilla, Aptdo. 1160, 41080 Sevilla, Spain, E-mails: blanca@numer.us.es, cara@numer.us.es. 


\section{Introduction}

Let us consider a fluid in a domain $\mathcal{O}_{\varepsilon}$, limited at the bottom by a plane wall $\mathcal{P}$ and at the top by a wall $\mathcal{R}_{\varepsilon}$. We assume that $\mathcal{P}$ moves at a constant velocity while $\mathcal{R}_{\varepsilon}$ is at rest. The latter is assumed to consist of a smooth wall $\mathcal{R}$ covered with periodically distributed asperities of small size $\varepsilon$. We are then concerned with the asymptotic behaviour, as $\varepsilon \rightarrow 0$, of the velocity and the pressure in the fluid.

The case in which the fluid adheres to the walls has been considered in [?], [?] and [?]. In [?], [?], the wall $\mathcal{R}$ is a plate. Using boundary layer correctors, it is proved that, outside a neighbourhood of the rugose zone, the flow behaves asymptotically as a Couette flow, up to an exponentially small error. An accurate approximation of the drag is given which shows that there is no palpable drag reduction. These results have been then extended to the case of a flow governed by Navier-Stokes equations, see [?]. Let us also mention a recent paper by W. Jäger and A. Mikelić [?] on the laminar viscous channel flow, with the lateral surface of the channel containing surface irregularities. The fluid satisfies a no-slip boundary condition on the rugose surface and it is supposed that a uniform pressure gradient is maintened in the longitudinal direction in the channel. So the limit flow is a Hagen-Poiseuille flow. Using the correponding boundary layers, the authors derive a wall law which gives an approximation of the tangential drag force at order $O\left(\varepsilon^{3 / 2}\right)$.

As appears from the quoted works, if the fluid is assumed to adhere to the walls, that is to say, if Dirichlet conditions are imposed, then the effect of rugosity is negligible. More precisely, the drag, the velocity field, and the pressure related to $\mathcal{R}_{\varepsilon}$ converge to those related to $\mathcal{R}$ as $\varepsilon \rightarrow 0$.

In this paper, we assume that, on the walls, the fluid satisfies conditions of Fourier kind. In particular, on the rugose wall we impose

$$
\sigma \cdot n+k u=0 \text { on } \mathcal{R}_{\varepsilon}
$$

where $\sigma$ is the usual stress tensor and $k$ is a friction coefficient. We prove that these conditions, contrarily to Dirichlet (no-slip) conditions, bring a leading part to rugosity : as $\varepsilon \rightarrow 0$, the drag experienced by the rugose wall $\mathcal{R}_{\varepsilon}$ converges to the drag associated with the smooth wall $\mathcal{R}$ provided with an homogenized friction coefficient $K$ which is not constant and depends on the profile of asperities. We calculate the limit flow and we give estimates of the deviations of the drag, velocity field and pressure, in terms of the size $\varepsilon$ of the asperities. In the particular case of a plate, the limit drag is larger than the drag of the smooth wall, see Remark 2.1.

In the case of Laplace or Poisson equation with Fourier or Neumann boundary conditions, similar results to those of the present paper have been obtained by O.A. Oleinik, A.S. Shamaev and G.A. Yosifian [?] and by G.A. Chechkin, A. Friedman and A.L. Piatnitski [?]. See also E. Sanchez-Palencia [?]. 
In fact, it is not completely realistic to assume that a fluid satisfies (??), unless the wall $\mathcal{R}_{\varepsilon}$ has some kind of porosity, see R.L. Panton [?, p. 149-152]. It would be more appropriate, instead, to impose the slip conditions

$$
u \cdot n=0, \quad(\sigma \cdot n)_{\operatorname{tang}}+k u=0 \text { on } \mathcal{R}_{\varepsilon},
$$

where $(\sigma \cdot n)_{\operatorname{tang}}$ denotes the tangential component of $\sigma \cdot n$. However, (??) may be used for large $k$ as a formal approximation of the Dirichlet condition $u=0$.

It would be very interesting to extend the present results to the case in which conditions like (??) are considered, but there are some technical difficulties to do this. Our results may be considered as a first step in this direction. Let us mention here that slip boundary conditions have been considered by G. Allaire [?], and D. Cioranescu, P. Donato and H. I. Ene [?] for homogenization of Stokes or Navier-Stokes equations in domains containing periodically distributed obstacles. For conditions of Fourier kind, we refer to C. Conca [?], [?].

\section{The main result}

The smooth wall $\mathcal{R}$ is assumed to be the graph of a function $r$ on $\mathbb{R}^{2}$, with

$$
r \text { is Lipschitz-continuous, positive and }\left(l_{1}, l_{2}\right) \text {-periodic }
$$

(the latter means that $r$ is periodic with respect to $x_{i}$ with period $l_{i}$ for $i=1$ or 2 ). For each $\varepsilon>0$, the rugose wall $\mathcal{R}_{\varepsilon}$ is assumed to be the graph of the function $r_{\varepsilon}$ defined on $\mathbb{R}^{2}$ as follows : for any $x^{\prime}=\left(x_{1}, x_{2}\right)$,

$$
r_{\varepsilon}\left(x^{\prime}\right)=r\left(x^{\prime}\right)\left(1+\varepsilon \eta\left(x^{\prime}, \frac{x^{\prime}}{\varepsilon}\right)\right) \text {. }
$$

Here, $\eta=\eta\left(x^{\prime}, y^{\prime}\right)$ is a function on $\mathbb{R}^{2} \times \mathbb{R}^{2}$ satisfying

$\eta$ is Lipschitz-continuous and $l_{1}, l_{2}$-periodic with respect to $x^{\prime}$ and $y^{\prime}$.

In order to ensure that $r_{\varepsilon}$ satisfies (??), we assume that

$$
\varepsilon\|\eta\|_{L^{\infty}\left(S^{2}\right)} \leq \frac{1}{2}, \quad \frac{1}{\varepsilon} \text { is an integer number. }
$$

The fluid occupies the unbounded domain

$$
\mathcal{O}_{\varepsilon}=\left\{x \in \mathbb{R}^{3}: x^{\prime} \in \mathbb{R}^{2}, 0<x_{3}<r_{\varepsilon}\left(x^{\prime}\right)\right\} .
$$

Setting $S=\left(0, l_{1}\right) \times\left(0, l_{2}\right), \mathcal{O}_{\varepsilon}$ can be viewed as generated by periodic translations of the bounded domain

$$
\Omega_{\varepsilon}=\left\{x \in \mathbb{R}^{3}: x^{\prime} \in S, 0<x_{3}<r_{\varepsilon}\left(x^{\prime}\right)\right\} .
$$


Observe that $\partial \Omega_{\varepsilon}$ consists of the following parts of the walls $\mathcal{R}_{\varepsilon}$ and $\mathcal{P}$

$$
R_{\varepsilon}=\left\{x \in \mathbb{R}^{3}: x^{\prime} \in S, x_{3}=r_{\varepsilon}\left(x^{\prime}\right)\right\}, \quad P=\left\{x \in \mathbb{R}^{3}: x^{\prime} \in S, x_{3}=0\right\}
$$

and the lateral immaterial boundary

$$
L=\left\{x \in \mathbb{R}^{3}: x^{\prime} \in \partial S, 0 \leq x_{3} \leq r_{\varepsilon}\left(x^{\prime}\right)\right\} .
$$

For each $m \geq 0$, let us introduce the space

$$
\begin{aligned}
& H_{\mathrm{per}}^{m}\left(\Omega_{\varepsilon}\right)=\left\{v \in H_{\mathrm{loc}}^{m}\left(\mathcal{O}_{\varepsilon}\right): v \in H^{m}\left(\Omega_{\varepsilon}\right)\right. \\
&\left.v\left(x+\left(l_{1}, 0,0\right)\right)=v\left(x+\left(0, l_{2}, 0\right)\right)=v(x) \text { for a.e. } x \in \mathcal{O}_{\varepsilon}\right\} .
\end{aligned}
$$

In other words, $H_{\text {per }}^{m}\left(\Omega_{\varepsilon}\right)$ is formed by all $\left(l_{1}, l_{2}\right)$-periodic functions on $\mathcal{O}_{\varepsilon}$ which are $H^{m}$ in any bounded subset (and not only in compact subsets, as $H_{\mathrm{loc}}^{m}$ means). As usual, for $m=0$, we write $L_{\text {per }}^{2}\left(\Omega_{\varepsilon}\right)$.

The velocity field and the pressure are assumed to satisfy

$$
\left\{\begin{array}{l}
u_{\varepsilon} \in\left(H_{\mathrm{per}}^{1}\left(\Omega_{\varepsilon}\right)\right)^{3}, \quad p_{\varepsilon} \in L_{\mathrm{per}}^{2}\left(\Omega_{\varepsilon}\right), \\
-\nu \Delta u_{\varepsilon}+\nabla p_{\varepsilon}=0, \quad \nabla \cdot u_{\varepsilon}=0 \text { in } \mathcal{O}_{\varepsilon}, \\
\sigma_{\varepsilon} \cdot n+k u=0 \text { on } \mathcal{R}_{\varepsilon}, \\
\sigma_{\varepsilon} \cdot n+k(u-g)=0 \text { on } \mathcal{P},
\end{array}\right.
$$

where $\nu>0$ is the viscosity,

$$
\sigma_{\varepsilon}=\sigma\left(u_{\varepsilon}, p_{\varepsilon}\right)=-p_{\varepsilon} \operatorname{Id}+\nu\left(\nabla u_{\varepsilon}+{ }^{t} \nabla u_{\varepsilon}\right),
$$

$n$ is the outwards unit normal vector field, $k>0$ is a friction coefficient and $g=$ $\left(g^{\prime}, 0\right)$ is the velocity of the wall $\mathcal{P}$.

The existence and uniqueness of a solution is proved in proposition 3.1. Remark that the boundary conditions on $\mathcal{R}_{\varepsilon}$ and $\mathcal{P}$ are meaningful, since (??) gives $\sigma_{\varepsilon} \in$ $\left(L_{\text {per }}^{2}\left(\Omega_{\varepsilon}\right)\right)^{3 \times 3}$ and

$$
\nabla \cdot \sigma_{\varepsilon}=-\nabla p_{\varepsilon}+\nu \Delta u_{\varepsilon}+{ }^{t} \nabla\left(\nabla \cdot u_{\varepsilon}\right)=0
$$

This allows to define the normal trace $\sigma_{\varepsilon} \cdot n$ in $\left(H_{\mathrm{loc}}^{-1 / 2}\left(\partial \mathcal{O}_{\varepsilon}\right)\right)^{3}$.

The hydrodynamical drag $T_{\varepsilon}$ associated with the bounded part $R_{\varepsilon}$ of the wall $\mathcal{R}_{\varepsilon}$ (the drag of the whole wall is infinite) is by definition the projection of the force exerted on $R_{\varepsilon}$ by the fluid, that is

$$
T_{\varepsilon}=-g \cdot \int_{R_{\varepsilon}} \sigma_{\varepsilon} \cdot n d s
$$


Thanks to the boundary condition on $\mathcal{R}_{\varepsilon}$, it reads as well

$$
T_{\varepsilon}=g \cdot \int_{R_{\varepsilon}} u_{\varepsilon} d s
$$

Let us now define the homogenized friction coefficient $K=K\left(x^{\prime}, r\left(x^{\prime}\right)\right)$. First, we introduce the following function $m \in L^{\infty}\left(\mathbb{R}^{2} \times \mathbb{R}^{2}\right)$ : for any $\left(x^{\prime}, y^{\prime}\right) \in \mathbb{R}^{2} \times \mathbb{R}^{2}$,

$$
m\left(x^{\prime}, y^{\prime}\right)=\left(\frac{1+\left|\nabla r\left(x^{\prime}\right)+r\left(x^{\prime}\right) \nabla_{y^{\prime}} \eta\left(x^{\prime}, y^{\prime}\right)\right|^{2}}{1+\left|\nabla r\left(x^{\prime}\right)\right|^{2}}\right)^{\frac{1}{2}} \text {. }
$$

Then we put, for all $x^{\prime} \in \mathbb{R}^{2}$,

$$
\langle m\rangle\left(x^{\prime}\right)=\frac{1}{|S|} \int_{S} m\left(x^{\prime}, y^{\prime}\right) d y^{\prime}
$$

Obviously, we have $\langle m\rangle \in L^{\infty}\left(\mathbb{R}^{2}\right)$. The homogenized friction coefficient is given $\mathcal{R}$ as follows : for all $x^{\prime} \in \mathbb{R}^{2}$,

$$
K\left(x^{\prime}, r\left(x^{\prime}\right)\right)=k\langle m\rangle\left(x^{\prime}\right) .
$$

It is therefore a $\left(l_{1}, l_{2}\right)$-periodic function which belongs to $L^{\infty}(\mathcal{R})$.

We will prove that $\left(u_{\varepsilon}, p_{\varepsilon}\right)$ converges in an appropriate sense to the unique solution of the system

$$
\left\{\begin{array}{l}
u_{0} \in\left(H_{\mathrm{per}}^{1}(\Omega)\right)^{3}, \quad p_{0} \in L_{\mathrm{per}}^{2}(\Omega), \\
-\nu \Delta u_{0}+\nabla p_{0}=0, \quad \nabla \cdot u_{0}=0 \quad \text { in } \mathcal{O}, \\
\sigma_{0} \cdot n+K u_{0}=0 \text { on } \mathcal{R}, \\
\sigma_{0} \cdot n+k\left(u_{0}-g\right)=0 \text { on } \mathcal{P},
\end{array}\right.
$$

where $\sigma_{0}=-p_{0} \operatorname{Id}+\nu\left(\nabla u_{0}+{ }^{t} \nabla u_{0}\right), \mathcal{R}$ is the graph of $r, \mathcal{O}$ is the domain bounded by $\mathcal{R}$ and $\mathcal{P}$ and $\Omega=\left\{x \in \mathbb{R}^{3}: x^{\prime} \in S, 0<x_{3}<r\left(x^{\prime}\right)\right\}$. Furthermore, we will prove that the limit drag is

$$
T_{0}=-g \cdot \int_{R} \sigma_{0} \cdot n d s=g \cdot \int_{R} K u_{0} d s .
$$

Notice that, in general, $u_{0}$ is not the velocity field related to the smooth wall $\mathcal{R}$ because $K \neq k$, and $T_{0}$ is not the drag experienced by $R$.

Since the domain $\Omega_{\varepsilon}$ varies with $\varepsilon$, the convergence of $u_{\varepsilon}$ and $p_{\varepsilon}$ cannot hold in the whole domain $\Omega$. We will obtain convergence outside a neighbourhood of $\mathcal{R}$ of arbitrary small size $\delta>0$, that is, in all subdomains of the form

$$
\omega_{\delta}=\left\{x \in \mathbb{R}^{3}: x^{\prime} \in S, 0<x_{3}<r\left(x^{\prime}\right)-\delta\right\} .
$$

Our main result is the following. 
Theorem 2.1 Assume $r \in W^{3, \infty}\left(\mathbb{R}^{2}\right)$. There exists $C>0$ such that, for any $\varepsilon>0$ satisfying (??), we have :

$$
\left|T_{\varepsilon}-T_{0}\right| \leq C \sqrt{\varepsilon}
$$

Moreover, for any $\delta>0$ there exists $C_{\delta}>0$ such that, for any $\varepsilon$ satisfying (??), we have:

$$
\begin{aligned}
\left\|u_{\varepsilon}-u_{0}\right\|_{H^{1}\left(\omega_{\delta}\right)} & \leq C_{\delta} \sqrt{\varepsilon} \\
\left\|p_{\varepsilon}-p_{0}\right\|_{L^{2}\left(\omega_{\delta}\right)} & \leq C_{\delta} \sqrt{\varepsilon}
\end{aligned}
$$

Remark 2.1 The drag $T_{\varepsilon}$ of a rugose plate is strictly greater than the drag $T$ of the corresponding (homogenized) smooth plate. Indeed, assume that $r\left(x^{\prime}\right) \equiv l_{3}$ (a positive real number) and $\eta$ depends only on $y^{\prime}$. Then $\langle m\rangle$ is independent of $x^{\prime}$ and is greater than 1 unless $\eta$ is a constant. Accordingly, we have

$$
\lim _{\varepsilon \rightarrow 0} T_{\varepsilon}=T_{0}=\frac{\nu l_{1} l_{2} k\langle m\rangle|g|^{2}}{\nu(1+\langle m\rangle)+l_{3} k\langle m\rangle}>T=\frac{\nu l_{1} l_{2} k|g|^{2}}{2 \nu+l_{3} k} .
$$

Remark 2.2 Notice that

$$
\frac{1}{1+\left|\nabla r\left(x^{\prime}\right)\right|^{2}} \leq\langle m\rangle\left(x^{\prime}\right) \leq\left(1+\frac{\left(r\left(x^{\prime}\right)\right)^{2}}{1+\left|\nabla r\left(x^{\prime}\right)\right|^{2}} \frac{1}{|S|} \int_{S}\left|\nabla_{y^{\prime}} \eta\left(x^{\prime}, y^{\prime}\right)\right| d y^{\prime}\right)^{1 / 2} .
$$

The last quantity is bounded from above by the local asymptotic ratio of the $R_{\varepsilon}$-area and the $R$-area. In other words, for any $x^{\prime} \in S$, we have

$$
\left\langle m^{\prime}\right\rangle\left(x^{\prime}\right) \leq \frac{\lim _{a \rightarrow 0} \lim _{\varepsilon \rightarrow 0}\left|R_{\varepsilon} \cup B\left(x^{\prime} ; a\right)\right|}{\lim _{a \rightarrow 0}\left|R \cup B\left(x^{\prime} ; a\right)\right|}
$$

where $B\left(x^{\prime} ; a\right)$ is the ball centered at $\left(x^{\prime}, r\left(x^{\prime}\right)\right)$ of radius $a$.

Remark 2.3 The drag $T_{\varepsilon}$ can also be written in the form

$$
T_{\varepsilon}=g \cdot \int_{P} \sigma_{\varepsilon} \cdot n d s=-k g \cdot \int_{P}\left(u_{\varepsilon}-g\right) d s .
$$

Indeed, the following equalities hold :

$$
\begin{gathered}
-g \cdot \int_{R_{\varepsilon}} \sigma_{\varepsilon} \cdot n d s=-g \cdot \int_{R_{\varepsilon} \cup P} \sigma_{\varepsilon} \cdot n d s+g \cdot \int_{P} \sigma_{\varepsilon} \cdot n d s \\
=-g \cdot \int_{\Omega_{\varepsilon}} \nabla \cdot \sigma_{\varepsilon} d s+g \cdot \int_{P} \sigma_{\varepsilon} \cdot n d s .
\end{gathered}
$$

A similar equality holds for $T_{0}$. 


\section{Existence, uniqueness and estimates}

We will prove that there exists exactly one solution $\left(u_{\varepsilon}, p_{\varepsilon}\right)$ to (??) and exactly one solution $\left(u_{0}, p_{0}\right)$ to (??). Notice that (??) is similar to (??) with a varying friction coefficient, since both walls $\mathcal{R}_{\varepsilon}$ and $\mathcal{R}$ are the graphs of periodic Lipschitz functions. Therefore, in order to put these two problems in the same framework, we will assume in this Section that

$$
k \in L_{\mathrm{per}}^{\infty}\left(\Omega_{\varepsilon}\right), \quad k \geq \kappa>0,
$$

where $\kappa$ is a real number.

We will use the following variational formulation :

$$
\left\{\begin{array}{l}
u_{\varepsilon} \in\left(H_{\mathrm{per}}^{1}\left(\Omega_{\varepsilon}\right)\right)^{3}, \quad p_{\varepsilon} \in L_{\mathrm{per}}^{2}\left(\Omega_{\varepsilon}\right), \\
2 \nu \int_{\Omega_{\varepsilon}} e\left(u_{\varepsilon}\right): e(\varphi)-\int_{\Omega_{\varepsilon}} p_{\varepsilon} \nabla \cdot \varphi+\int_{R_{\varepsilon} \cup P} k u_{\varepsilon} \cdot \varphi=\int_{P} k g \cdot \varphi \quad \forall \varphi \in\left(H_{\mathrm{per}}^{1}\left(\Omega_{\varepsilon}\right)\right)^{3}, \\
\nabla \cdot u_{\varepsilon}=0
\end{array}\right.
$$

where $e(\varphi)=\frac{1}{2}\left(\nabla \varphi+{ }^{t} \nabla \varphi\right)$ and $e(u): e(\varphi)=\sum_{i, j} e_{i j}(u) e_{i j}(\varphi)$.

Proposition 3.1 Problem (??) is equivalent to (??) and possesses exactly one solution. Furthermore, one has

$$
\left\|u_{\varepsilon}\right\|_{H^{1}\left(\Omega_{\varepsilon}\right)}+\left\|p_{\varepsilon}\right\|_{L^{2}\left(\Omega_{\varepsilon}\right)} \leq C,
$$

where $C$ is independent of $\varepsilon$.

Obviously, this result also provides the existence and uniqueness of a solution $\left(u_{0}, p_{0}\right)$ to $(? ?)$.

In order to prove this proposition, we need some previous results. In particular, we need a Korn inequality for a special class of star-shaped domains. By definition, $D$ is star-shaped, with respect to a ball $B$ if the segment connecting any two points $x \in B$ and $y \in D$ lies in $D$.

Lemma 3.1 There exists $C>0$ such that, for any bounded domain $D \subset \mathbb{R}^{3}$ of diameter $R$ which is star-shaped with respect to a ball $B$ of radius $\rho$ and for any $v \in\left(H^{1}(D)\right)^{3}$, the following inequality holds

$$
\|\nabla v\|_{L^{2}(D)}^{2} \leq C\left(\frac{R}{\rho}\right)^{3}\left(\|e(v)\|_{L^{2}(D)}^{2}+\|\nabla v\|_{L^{2}(B)}^{2}\right) .
$$


For the proof see O.A. Oleinik, A.S. Shamaev and G.A. Yosifian, [?, Theorem 2.10, p. 23].

Let us put

$$
D_{\star}=\left\{x \in \mathbb{R}^{3}: x^{\prime} \in S, 0<x_{3}<\frac{l_{3}}{2}\right\} .
$$

Lemma 3.2 There exists $C>0$, only depending on $S$ and $l_{3}$, such that, for all $v \in\left(H^{1}\left(D_{\star}\right)\right)^{3}$,

$$
\|\nabla v\|_{L^{2}\left(D_{\star}\right)}^{2} \leq C\left(\|e(v)\|_{L^{2}\left(D_{\star}\right)}^{2}+\int_{P}|v|^{2} d s\right) .
$$

Proof: Suppose the assertion in this lemma is false. Then, for each $m \geq 1$, there exists $w_{m} \in\left(H^{1}\left(D_{\star}\right)\right)^{3}$ such that

$$
\left\|\nabla w_{m}\right\|_{L^{2}\left(D_{\star}\right)}^{2}>m\left(\left\|e\left(w_{m}\right)\right\|_{L^{2}\left(D_{\star}\right)}^{2}+\int_{P}\left|w_{m}\right|^{2} d s\right) .
$$

Let us put $v_{m}=w_{m} /\left\|\nabla w_{m}\right\|_{L^{2}\left(D_{\star}\right)}$. Then $\left\|\nabla v_{m}\right\|_{L^{2}\left(D_{\star}\right)}=1$ and

$$
\left\|e\left(v_{m}\right)\right\|_{L^{2}\left(D_{\star}\right)}^{2}+\int_{P}\left|v_{m}\right|^{2}<\frac{1}{m}
$$

whence we obtain the following as $m \rightarrow \infty$ :

$$
e\left(v_{m}\right) \rightarrow 0 \text { in }\left(L^{2}\left(D_{\star}\right)\right)^{3 \times 3}, \quad \int_{P}\left|v_{m}\right|^{2} d s \rightarrow 0 .
$$

On the other hand, the following estimate holds for all $v \in\left(H^{1}\left(D_{\star}\right)\right)^{3}$ :

$$
\int_{D_{\star}}|v|^{2} d s \leq l_{3}\left(\int_{P}|v|^{2}+\frac{l_{3}}{2} \int_{D_{\star}}|\nabla v|^{2}\right) .
$$

Indeed, for any regular $v$ and any $x=\left(x^{\prime}, x_{3}\right)$ in $D_{\star}$, one has

$$
v(x)=v\left(x^{\prime}, 0\right)+\int_{0}^{x_{3}} \partial_{x_{3}} v\left(x^{\prime}, y_{3}\right) d y_{3} .
$$

Consequently,

$$
|v(x)|^{2} \leq 2\left|v\left(x^{\prime}, 0\right)\right|^{2}+2\left(\int_{0}^{l_{3} / 2}\left|\partial_{x_{3}} v\left(x^{\prime}, y_{3}\right)\right| d y_{3}\right)^{2}
$$

whence

$$
\int_{0}^{l_{3} / 2}\left|v\left(x^{\prime}, x_{3}\right)\right|^{2} d x_{3} \leq l_{3}\left(\left|v\left(x^{\prime}, 0\right)\right|^{2}+\left(\int_{0}^{l_{3} / 2}\left|\partial_{x_{3}} v\left(x^{\prime}, y_{3}\right)\right| d y_{3}\right)^{2}\right)
$$


and

$$
\begin{aligned}
& \int_{S} \int_{0}^{l_{3} / 2}\left|v\left(x^{\prime}, x_{3}\right)\right|^{2} d x_{3} d x^{\prime} \\
& \quad \leq l_{3}\left(\int_{P}\left|v\left(x^{\prime}, 0\right)\right|^{2} d x^{\prime}+\frac{1}{2} \int_{S} \int_{0}^{l_{3} / 2}\left|\partial_{x_{3}} v\left(x^{\prime}, y_{3}\right)\right|^{2} l_{3} d y_{3} d x^{\prime}\right) .
\end{aligned}
$$

This proves (??), at least when $v$ is regular enough. By density, (??) holds for all $v$ in $\left(H^{1}\left(D_{\star}\right)\right)^{3}$. It follows from (??) that $v_{m}$ is uniformly bounded in $\left(H^{1}\left(D_{\star}\right)\right)^{3}$. From the compactness of the embedding $H^{1}\left(D_{\star}\right) \hookrightarrow L^{2}\left(D_{\star}\right)$, there exists a subsequence, still denoted $v_{m}$, that converges strongly in $\left(L^{2}\left(D_{\star}\right)\right)^{3 \times 3}$ to some $v \in\left(H^{1}\left(D_{\star}\right)\right)^{3}$. In view of Korn inequality in Lipschitz domains, one has

$$
\left\|v_{m^{\prime}}-v_{m}\right\|_{H^{1}\left(D_{\star}\right)}^{2} \leq C\left(\left\|e\left(v_{m^{\prime}}\right)-e\left(v_{m}\right)\right\|_{L^{2}\left(D_{\star}\right)}^{2}+\left\|v_{m^{\prime}}-v_{m}\right\|_{L^{2}\left(D_{\star}\right)}^{2}\right),
$$

where the constant $C$ depends only on $S$ and $l_{3}$, see [?], [?]. These inequalities and (??) show that $v_{m}$ converges strongly in $H^{1}\left(D_{\star}\right)$ to $v$ and, also, that

$$
\|\nabla v\|_{L^{2}\left(D_{\star}\right)}=1, \quad\|e(v)\|_{L^{2}\left(D_{\star}\right)}=0, \quad \int_{P}|v|^{2} d s=0 .
$$

But the equality $e(v)=0$ implies that $v$ is a rigid displacement, i.e. $v=A x+b$ where $A$ is a skew-symmetric constant matrix and $b$ is a constant vector. This fact, together with the third equality in (??), implies $v=0$. This leads to a contradiction and proves the lemma.

Lemma 3.3 Let $\varepsilon$ satisfy (??). Then, for any $v \in\left(H^{1}\left(\Omega_{\varepsilon}\right)\right)^{3}$,

$$
\|v\|_{H^{1}\left(\Omega_{\varepsilon}\right)}^{2} \leq C\left(\|e(v)\|_{L^{2}\left(\Omega_{\varepsilon}\right)}^{2}+\int_{P}|v|^{2}\right)
$$

where $C$ depends only of $l_{1}, l_{2}, r$ and $\eta$.

Proof: The function $r_{\varepsilon}$ defined by (??) is Lipschitz-continous, with a Lipschitz constant independent of $\varepsilon$. It is also bounded from below by a positive number independent of $\varepsilon$. Therefore, if $l_{1}$ and $l_{2}$ are small enough, there exists a ball $B$ independent of $\varepsilon$ such that $\Omega_{\varepsilon}$ is star-shaped with respect to $B$. Moreover, $B$ can be chosen in $D_{\star}$. For arbitrarily given $l_{1}$ and $l_{2}$, by dividing $S$ in sufficiently small squares, it follows that $\Omega_{\varepsilon}$ is the union of $m$ domains $\Omega_{\varepsilon}^{i}$ which are respectively starshaped with respect to the balls $B^{i}$, with $m$ and $B^{i}$ independent of $\varepsilon$. Lemma 3.1 yields the following for each $i$ and for all $v \in\left(H^{1}\left(\Omega_{\varepsilon}\right)\right)^{3}$ :

$$
\|\nabla v\|_{L^{2}\left(\Omega_{\varepsilon}^{i}\right)}^{2} \leq C\left(\|e(v)\|_{L^{2}\left(\Omega_{\varepsilon}^{i}\right)}^{2}+\|\nabla v\|_{L^{2}\left(B_{i}\right)}^{2}\right) .
$$

Adding these inequalities for $i=1, \ldots, m$, we find that

$$
\|\nabla v\|_{L^{2}\left(\Omega_{\varepsilon}\right)}^{2} \leq C\left(\|e(v)\|_{L^{2}\left(\Omega_{\varepsilon}\right)}^{2}+\|\nabla v\|_{L^{2}\left(D_{\star}\right)}^{2}\right)
$$


and using (??) we obtain

$$
\|\nabla v\|_{L^{2}\left(\Omega_{\varepsilon}\right)}^{2} \leq C\left(\|e(v)\|_{L^{2}\left(\Omega_{\varepsilon}\right)}^{2}+\int_{P}|v|^{2}\right) .
$$

It is also clear that

$$
\|v\|_{L^{2}\left(\Omega_{\varepsilon}\right)}^{2} \leq C\left(\|\nabla v\|_{L^{2}\left(\Omega_{\varepsilon}\right)}^{2}+\int_{P}|v|^{2}\right) .
$$

This can be seen arguing as in the proof of (??) in the previous lemma. From (??) and (??), we deduce (??) and the lemma is proved.

Lemma 3.4 Let $\varepsilon$ satisfy (??). For any $F \in L^{2}\left(\Omega_{\varepsilon}\right)$ and any $w_{0} \in\left(H^{1 / 2}\left(\partial \Omega_{\varepsilon}\right)\right)^{3}$ such that

$$
\int_{\Omega_{\varepsilon}} F=\int_{\partial \Omega_{\varepsilon}} w_{0} \cdot n
$$

there exists a function $w_{\varepsilon} \in\left(H^{1}\left(\Omega_{\varepsilon}\right)\right)^{3}$ such that

$$
\nabla \cdot w_{\varepsilon}=F \quad \text { in } \Omega_{\varepsilon}, \quad w_{\varepsilon}=w_{0} \text { on } \partial \Omega_{\varepsilon}
$$

and

$$
\left\|w_{\varepsilon}\right\|_{H^{1}\left(\Omega_{\varepsilon}\right)} \leq C\left(\left\|w_{0}\right\|_{H^{1 / 2}\left(\partial \Omega_{\varepsilon}\right)}+\|F\|_{L^{2}\left(\Omega_{\varepsilon}\right)}\right),
$$

where $C$ is independent of $F, w_{0}$ and $\varepsilon$.

The proof of this lemma is essentially contained in theorem 3.1 of [?, p. 116,124].

Lemma 3.5 Let $\varepsilon$ satisfy (??). For each $q \in L_{\text {per }}^{2}\left(\Omega_{\varepsilon}\right)$, there exists $z_{\varepsilon} \in\left(H_{\mathrm{per}}^{1}\left(\Omega_{\varepsilon}\right)\right)^{3}$ such that

$$
z_{\varepsilon}=0 \quad \text { on } R_{\varepsilon}, \quad \nabla \cdot z_{\varepsilon}=q \text { in } \Omega_{\varepsilon}, \quad\left\|z_{\varepsilon}\right\|_{H^{1}\left(\Omega_{\varepsilon}\right)} \leq C\|q\|_{L^{2}\left(\Omega_{\varepsilon}\right)},
$$

where $C$ is independent of $q$ and $\varepsilon$.

Proof: Let $v_{\varepsilon} \in\left(H^{1 / 2}\left(\partial \Omega_{\varepsilon}\right)\right)^{3}$ be such that $v_{\varepsilon}=0$ on $R_{\varepsilon}$,

$$
\int_{\partial \Omega_{\varepsilon}} v_{\varepsilon} \cdot n d s=\left|\Omega_{\varepsilon}\right|
$$

and $\left\|v_{\varepsilon}\right\|_{H^{1 / 2}\left(\partial \Omega_{\varepsilon}\right)}$ is bounded uniformly with respect to $\varepsilon$. It is clear that $v_{\varepsilon}$ can be chosen $x^{\prime}$-periodic, i.e. satisfiying

$$
v_{\varepsilon}\left(x_{1}, 0, x_{3}\right)=v_{\varepsilon}\left(x_{1}, l_{1}, x_{3}\right), \quad v_{\varepsilon}\left(0, x_{2}, x_{3}\right)=v_{\varepsilon}\left(l_{1}, x_{2}, x_{3}\right) .
$$

Using lemma 3.4 with $F \equiv 1$ and $w_{0}=v_{\varepsilon}$, we obtain a function $\bar{v}_{\varepsilon} \in\left(H^{1}\left(\Omega_{\varepsilon}\right)\right)^{3}$ such that

$$
\nabla \cdot \bar{v}_{\varepsilon}=1 \text { in } \Omega_{\varepsilon}, \quad \bar{v}_{\varepsilon}=v_{\varepsilon} \text { on } \partial \Omega_{\varepsilon}
$$


and

$$
\left\|\bar{v}_{\varepsilon}\right\|_{H^{1}\left(\Omega_{\varepsilon}\right)} \leq C\left(\left\|v_{\varepsilon}\right\|_{H^{1 / 2}\left(\partial \Omega_{\varepsilon}\right)}+\left|\Omega_{\varepsilon}\right|^{1 / 2}\right) \leq C .
$$

Obviously, since $v_{\varepsilon}$ is $x^{\prime}$-periodic, the same is true for $\bar{v}_{\varepsilon}$. Let $q$ be an arbitrary function of $L_{\text {per }}^{2}\left(\Omega_{\varepsilon}\right)$. We can write $q$ in the form

$$
q=p+\frac{1}{\left|\Omega_{\varepsilon}\right|} \int_{\Omega_{\varepsilon}} q
$$

with $p \in L^{2}\left(\Omega_{\varepsilon}\right)$ and $\int_{\Omega_{\varepsilon}} p=0$. By lemma 3.4 with $w_{0}=0$, there exists $w_{\varepsilon} \in$ $\left(H_{0}^{1}\left(\Omega_{\varepsilon}\right)\right)^{3}$ such that

$$
\nabla \cdot w_{\varepsilon}=p \text { in } \Omega_{\varepsilon}, \quad\left\|w_{\varepsilon}\right\|_{H^{1}\left(\Omega_{\varepsilon}\right)} \leq C\|p\|_{L^{2}\left(\Omega_{\varepsilon}\right)},
$$

where $C$ is independent of $\varepsilon$ and $p$. Consequently, we have

$$
\left\|w_{\varepsilon}\right\|_{H^{1}\left(\Omega_{\varepsilon}\right)} \leq C\|q\|_{L^{2}\left(\Omega_{\varepsilon}\right)} .
$$

Let us put

$$
z_{\varepsilon}=w_{\varepsilon}+\left(\frac{1}{\left|\Omega_{\varepsilon}\right|} \int_{\Omega_{\varepsilon}} q\right) \bar{v}_{\varepsilon} .
$$

It is then clear from (??), (??) and (??) that $z_{\varepsilon} \in\left(H_{\mathrm{per}}^{1}\left(\Omega_{\varepsilon}\right)\right)^{3}, \nabla \cdot z_{\varepsilon}=q$ and

$$
\left\|z_{\varepsilon}\right\|_{H^{1}\left(\Omega_{\varepsilon}\right)} \leq C\|q\|_{L^{2}\left(\Omega_{\varepsilon}\right)}+\frac{C}{\left|\Omega_{\varepsilon}\right|}\left|\int_{\Omega_{\varepsilon}} q\right| \leq C\|q\|_{L^{2}\left(\Omega_{\varepsilon}\right)} .
$$

This proves the lemma.

Proof of Proposition 3.1: Let us first prove that (??) is equivalent to (??). Given a solution $\left(u_{\varepsilon}, p_{\varepsilon}\right)$ to (??), a normal trace $\sigma_{\varepsilon} \cdot n$ in $\left(H_{\text {loc }}^{-1 / 2}\left(\partial \mathcal{O}_{\varepsilon}\right)\right)^{3}$ is defined, thanks to (??), by : for all $\varphi \in\left(H^{1}\left(\Omega_{\varepsilon}\right)\right)^{3}$,

$$
\left\langle\sigma_{\varepsilon} \cdot n, \varphi\right\rangle_{\partial \Omega_{\varepsilon}}=\int_{\Omega_{\varepsilon}} \sigma_{\varepsilon} \cdot \nabla \varphi
$$

If in addition $\varphi$ is periodic, that is if $\varphi \in\left(H_{\text {per }}^{1}\left(\Omega_{\varepsilon}\right)\right)^{3}$, this reduces to

$$
\left\langle\sigma_{\varepsilon} \cdot n, \varphi\right\rangle_{R_{\varepsilon} \cup P}=\int_{\Omega_{\varepsilon}} \sigma_{\varepsilon} \cdot \nabla \varphi .
$$

Indeed, the contribution of the lateral part $L$ of the boundary is then $\left\langle\sigma_{\varepsilon} \cdot n, \varphi\right\rangle_{L}=0$ since, on opposite sides, $\sigma_{\varepsilon}$ and $\varphi$ are preserved while $n$ is replaced by the opposite vector. Using the boundary conditions and the following identity

$$
\sigma_{\varepsilon} \cdot \nabla \varphi=2 \nu e\left(u_{\varepsilon}\right): e(\varphi)-p_{\varepsilon} \nabla \cdot \varphi
$$


we obtain the equation in (??).

Conversely, let $\left(u_{\varepsilon}, p_{\varepsilon}\right)$ satisfy (??). Choosing $\varphi$ to be the periodic extension of a function in $\left(\mathcal{D}\left(\Omega_{\varepsilon}\right)\right)^{3}$ and using (??), we get $\int_{\Omega_{\varepsilon}} \sigma_{\varepsilon} \cdot \nabla \varphi=0$ and thus $\nabla \cdot \sigma_{\varepsilon}=0$. Then (??) holds and (??) reads, for all $\varphi \in\left(H_{\mathrm{per}}^{1}\left(\Omega_{\varepsilon}\right)\right)^{3}$,

$$
\left\langle\sigma_{\varepsilon} \cdot n, \varphi\right\rangle_{R_{\varepsilon} \cup P}+\int_{R_{\varepsilon} \cup P} k u_{\varepsilon} \cdot \varphi d s=\int_{P} k g \cdot \varphi d s .
$$

This provides the boundary conditions in (??); Stokes equation follows from $\nabla \cdot \sigma_{\varepsilon}=$ $0, \nabla \cdot u_{\varepsilon}=0$ and (??). Therefore (??) is equivalent to (??).

Let us now prove the existence and uniqueness of a solution $\left(u_{\varepsilon}, p_{\varepsilon}\right)$ to (??). We can put (??) in the form

$$
\left\{\begin{array}{l}
\left(u_{\varepsilon}, p_{\varepsilon}\right) \in\left(H_{\mathrm{per}}^{1}\left(\Omega_{\varepsilon}\right)\right)^{3} \times L_{\mathrm{per}}^{2}\left(\Omega_{\varepsilon}\right), \\
a\left(u_{\varepsilon}, \varphi\right)+b\left(\varphi, p_{\varepsilon}\right)=\int_{P} k \cdot g \varphi \quad \forall \varphi \in\left(H_{\mathrm{per}}^{1}\left(\Omega_{\varepsilon}\right)\right)^{3}, \\
b\left(u_{\varepsilon}, q\right)=0 \quad \forall q \in L_{\mathrm{per}}^{2}\left(\Omega_{\varepsilon}\right),
\end{array}\right.
$$

where the bilinear forms $a(\cdot, \cdot)$ and $b(\cdot, \cdot)$ are defined by

$$
a(u, v)=2 \nu \int_{\Omega_{\varepsilon}} e(u): e(v)+\int_{R_{\varepsilon} \cup P} k u \cdot v \quad \forall u, v \in\left(H_{\mathrm{per}}^{1}\left(\Omega_{\varepsilon}\right)\right)^{3}
$$

and

$$
b(v, q)=-\int_{\Omega_{\varepsilon}}(\nabla \cdot v) q \quad \forall u \in\left(H_{\mathrm{per}}^{1}\left(\Omega_{\varepsilon}\right)\right)^{3}, \forall q \in L_{\mathrm{per}}^{2}\left(\Omega_{\varepsilon}\right) .
$$

Taking into account Corollary 4.1, p. 61 of [?], in order to establish existence and uniqueness, it is sufficient to prove that $a(\cdot, \cdot)$ is coercive on $V_{\varepsilon}$, i.e.

$$
a(v, v) \geq \alpha\|v\|_{H^{1}\left(\Omega_{\varepsilon}\right)}^{2} \quad \forall v \in V_{\varepsilon}, \quad \alpha>0,
$$

and $b(\cdot, \cdot)$ satisfies the Brezzi-Babuska "inf-sup" condition

$$
\inf _{q \in L_{\mathrm{per}}^{2}\left(\Omega_{\varepsilon}\right), q \neq 0} \sup _{v \in\left(H_{\mathrm{per}}^{1}\left(\Omega_{\varepsilon}\right)\right)^{3}, v \neq 0} \frac{b(v, q)}{\|v\|_{H^{1}\left(\Omega_{\varepsilon}\right)}\|q\|_{L^{2}\left(\Omega_{\varepsilon}\right)}} \geq \beta>0 .
$$

In (??), $V_{\varepsilon}$ stands for the space

$$
V_{\varepsilon}=\left\{v \in\left(H_{\mathrm{per}}^{1}\left(\Omega_{\varepsilon}\right)\right)^{3}: b(v, q)=0 \quad \forall q \in L_{\mathrm{per}}^{2}\left(\Omega_{\varepsilon}\right)\right\} .
$$

Thus $V_{\varepsilon}$ is the space of all functions $v$ in $\left(H_{\text {per }}^{1}\left(\Omega_{\varepsilon}\right)\right)^{3}$ satisfying $\nabla \cdot v=0$ in $\Omega_{\varepsilon}$.

The inequality (??) holds for some $\alpha$ independent of $\varepsilon$. Indeed, thanks to lemma 3.3, we have :

$$
a(v, v)=2 \nu\|e(v)\|_{L^{2}\left(\Omega_{\varepsilon}\right)}^{2}+\int_{R_{\varepsilon} \cup P} k|v|^{2} \geq C\|v\|_{H^{1}\left(\Omega_{\varepsilon}\right)}^{2} .
$$


Let us now check the inf-sup condition (??). Assume that $q \in L_{\mathrm{per}}^{2}\left(\Omega_{\varepsilon}\right)$ and $q \neq 0$ and let $z_{\varepsilon}$ be the function furnished by lemma 3.5. Then

$$
\sup _{v \in\left(H_{\mathrm{per}}^{1}\left(\Omega_{\varepsilon}\right)\right)^{3}, v \neq 0} \frac{b(v, q)}{\|v\|_{H^{1}\left(\Omega_{\varepsilon}\right)}\|q\|_{L_{\mathrm{per}}^{2}\left(\Omega_{\varepsilon}\right)}} \geq \frac{-b\left(z_{\varepsilon}, q\right)}{\left\|z_{\varepsilon}\right\|_{H^{1}\left(\Omega_{\varepsilon}\right)}\|q\|_{L_{\mathrm{per}}^{2}\left(\Omega_{\varepsilon}\right)}} \geq \frac{1}{C}>0 .
$$

This proves (??). Since (??) and (??) hold, there exists exactly one pair $\left(u_{\varepsilon}, p_{\varepsilon}\right)$ which solves (??).

Let us estimate $u_{\varepsilon}$. Choosing $\varphi=u_{\varepsilon}$ in (??), we obtain

$$
a\left(u_{\varepsilon}, u_{\varepsilon}\right)=\int_{P} k g \cdot u_{\varepsilon}
$$

and then (??) gives

$$
\left\|u_{\varepsilon}\right\|_{H^{1}\left(\Omega_{\varepsilon}\right)} \leq C
$$

Finally, let us estimate $p_{\varepsilon}$. From lemma 3.5, there exists $w_{\varepsilon} \in\left(H_{\mathrm{per}}^{1}\left(\Omega_{\varepsilon}\right)\right)^{3}$ satisfying

$$
\nabla \cdot w_{\varepsilon}=p_{\varepsilon} \text { in } \Omega_{\varepsilon}, \quad\left\|w_{\varepsilon}\right\|_{H^{1}\left(\Omega_{\varepsilon}\right)} \leq C\left\|p_{\varepsilon}\right\|_{L^{2}\left(\Omega_{\varepsilon}\right)},
$$

where $C$ is independent of $\varepsilon, p_{\varepsilon}$ and $w_{\varepsilon}$. Let us choose $\varphi=w_{\varepsilon}$ in (??). Then we find

$$
\int_{\Omega_{\varepsilon}}\left|p_{\varepsilon}\right|^{2}=\nu \int_{\Omega_{\varepsilon}} e\left(u_{\varepsilon}\right): e\left(w_{\varepsilon}\right)+k \int_{P} u_{\varepsilon} \cdot w_{\varepsilon}-k \int_{P} g \cdot w_{\varepsilon} .
$$

The right-hand side can be bounded as follows :

$$
\begin{gathered}
\nu \int_{\Omega_{\varepsilon}} e\left(u_{\varepsilon}\right): e\left(w_{\varepsilon}\right)+k \int_{P} u_{\varepsilon} \cdot w_{\varepsilon}-k \int_{P} g \cdot w_{\varepsilon} \\
\leq C\left\|u_{\varepsilon}\right\|_{H^{1}\left(\Omega_{\varepsilon}\right)} \cdot\left\|w_{\varepsilon}\right\|_{H^{1}\left(\Omega_{\varepsilon}\right)}+C\left\|w_{\varepsilon}\right\|_{H^{1}\left(\Omega_{\varepsilon}\right)} \leq \frac{1}{2}\left\|p_{\varepsilon}\right\|_{L^{2}\left(\Omega_{\varepsilon}\right)}^{2}+C\left\|u_{\varepsilon}\right\|_{H^{1}\left(\Omega_{\varepsilon}\right)}+C .
\end{gathered}
$$

Thus, using (??), we deduce that

$$
\left\|p_{\varepsilon}\right\|_{L^{2}\left(\Omega_{\varepsilon}\right)} \leq C
$$

This ends the proof of proposition 3.1.

\section{The equations in the homogenized domain}

For any function $\psi=\psi\left(x^{\prime}, y^{\prime}\right)$ defined in $S \times S$ and periodic with respect to $y^{\prime}$, we will denote by $\bar{\psi}^{\varepsilon}$ the function defined on $S$ by

$$
\bar{\psi}^{\varepsilon}\left(x^{\prime}\right)=\psi\left(x^{\prime}, \frac{x^{\prime}}{\varepsilon}\right) .
$$


Let $\mathcal{L}_{\varepsilon}$ denote the one-to-one mapping from $\Omega$ onto $\Omega_{\varepsilon}$ defined by

$$
\mathcal{L}_{\varepsilon}\left(x^{\prime}, x_{3}\right)=\left(x^{\prime}, x_{3}\left(1+\varepsilon \eta\left(x^{\prime}, \frac{x^{\prime}}{\varepsilon}\right)\right)\right)=\left(x^{\prime}, x_{3}\left(1+\varepsilon \bar{\eta}^{\varepsilon}\left(x^{\prime}\right)\right)\right) .
$$

Since $\partial_{x_{i}} \bar{\eta}^{\varepsilon}={\overline{\partial_{x_{i}}}}^{\varepsilon}+\frac{1}{\varepsilon} \bar{\partial}_{y_{i}} \eta^{\varepsilon}$, the Jacobian matrix is

$$
\nabla \mathcal{L}_{\varepsilon}=\left(\begin{array}{ccc}
1 & 0 & x_{3}\left(\varepsilon \overline{\partial_{x_{1}} \eta}{ }^{\varepsilon}+{\overline{\partial_{y_{1}} \eta^{\varepsilon}}}^{\varepsilon}\right) \\
0 & 1 & x_{3}\left(\varepsilon \overline{\partial_{x_{2}} \eta}+\bar{\partial}_{y_{2}} \eta^{\varepsilon}\right. \\
0 & 0 & 1+\varepsilon \bar{\eta}^{\varepsilon}
\end{array}\right) .
$$

Given a function $v$ on $\Omega_{\varepsilon}$, we will denote by $\widehat{v}$ its image on $\Omega$, that is $\widehat{v}=v \circ \mathcal{L}_{\varepsilon}$. If $v \in L^{s}\left(\Omega_{\varepsilon}\right), 1 \leq s \leq \infty$, then $\widehat{v} \in L^{s}(\Omega)$ and

$$
\int_{\Omega_{\varepsilon}} v=\int_{\Omega} \widehat{v}\left(1+\varepsilon \bar{\eta}^{\varepsilon}\right)
$$

If $v \in W^{1, s}\left(\Omega_{\varepsilon}\right)$, then $\widehat{v} \in W^{1, s}(\Omega)$ and we have the following for all $i$ :

$$
\partial_{i} \widehat{v}=\sum_{j=1}^{3} \partial_{i} \mathcal{L}_{\varepsilon j} \widehat{\partial_{j} v}
$$

Conversely, for all $i$,

$$
\widehat{\partial_{i} v}=\sum_{j=1}^{3}\left(M_{\varepsilon}\right)_{i j} \partial_{j} \widehat{v}
$$

where

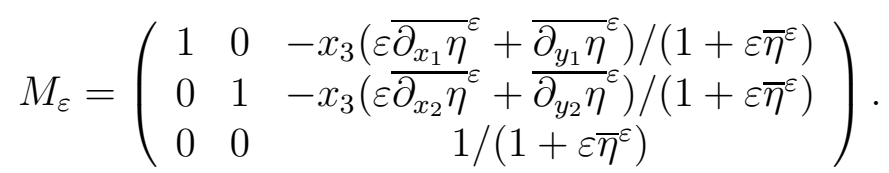

Another way to write (??) is the following :

$$
\widehat{\nabla v}=M_{\varepsilon} \nabla \widehat{v}
$$

Also,

$$
\widehat{\nabla \cdot v}={ }^{t} M_{\varepsilon}: \nabla \widehat{v}
$$

The image of the divergence can also be obtained from the dual formula

$$
\left(1+\varepsilon \bar{\eta}^{\varepsilon}\right) \widehat{\nabla \cdot v}=\nabla \cdot\left({ }^{t} N_{\varepsilon} \widehat{v}\right),
$$

where $N_{\varepsilon}=\left(1+\varepsilon \bar{\eta}^{\varepsilon}\right) M_{\varepsilon}$. Indeed, given $\psi \in \mathcal{D}\left(\Omega_{\varepsilon}\right)$, we have

$$
\begin{gathered}
\int_{\Omega}\left(1+\varepsilon \bar{\eta}^{\varepsilon}\right)(\widehat{\nabla \cdot v}) \widehat{\psi}=\int_{\Omega_{\varepsilon}}(\nabla \cdot v) \psi=-\int_{\Omega_{\varepsilon}} v \cdot \nabla \psi \\
=-\int_{\Omega}(\widehat{v} \cdot \widehat{\nabla \psi})\left(1+\varepsilon \bar{\eta}^{\varepsilon}\right)=-\int_{\Omega} \widehat{v} \cdot M_{\varepsilon} \nabla \widehat{\psi}\left(1+\varepsilon \bar{\eta}^{\varepsilon}\right) \\
=\int_{\Omega} \nabla \cdot\left(\left(1+\varepsilon \bar{\eta}^{\varepsilon}\right)^{t} M_{\varepsilon} \widehat{v}\right) \widehat{\psi} .
\end{gathered}
$$


Therefore, the weak formulation (??) (where $k>0$ is constant again) is equivalent to the following

$$
\begin{gathered}
\widehat{u}_{\varepsilon} \in\left(H_{\mathrm{per}}^{1}(\Omega)\right)^{3}, \quad \widehat{p}_{\varepsilon} \in L_{\mathrm{per}}^{2}(\Omega), \\
\left\{\begin{array}{c}
2 \nu \int_{\Omega}\left(\widehat{e}_{\varepsilon}\left(\widehat{u}_{\varepsilon}\right): \widehat{e}_{\varepsilon}(\varphi)\right) j_{\varepsilon}-\int_{\Omega} \widehat{p}_{\varepsilon} \nabla \cdot\left({ }^{t} N_{\varepsilon} \varphi\right)+k \int_{R}\left(\widehat{u}_{\varepsilon} \cdot \varphi\right) \mu_{\varepsilon} \\
+k \int_{P} \widehat{u}_{\varepsilon} \cdot \varphi=k \int_{P} g \cdot \varphi \quad \forall \varphi \in\left(H_{\mathrm{per}}^{1}(\Omega)\right)^{3}, \\
\nabla \cdot\left({ }^{t} N_{\varepsilon} \widehat{u}_{\varepsilon}\right)=0 .
\end{array}\right.
\end{gathered}
$$

Here, the following notations have been introduced :

$$
\begin{gathered}
\widehat{e}_{\varepsilon}(v)=\frac{1}{2}\left(M_{\varepsilon} \nabla v+{ }^{t}\left(M_{\varepsilon} \nabla v\right)\right), \\
j_{\varepsilon}=1+\varepsilon \bar{\eta}^{\varepsilon}, \quad \mu_{\varepsilon}=\frac{\left(1+\left|\nabla_{x^{\prime}} r_{\varepsilon}\right|^{2}\right)^{1 / 2}}{\left(1+\left|\nabla_{x^{\prime}}\right|^{2}\right)^{1 / 2}} .
\end{gathered}
$$

The asymptotic behavior of $\left(\widehat{u}_{\varepsilon}, \widehat{p}_{\varepsilon}\right)$ as $\varepsilon \rightarrow 0$ is explained in the following result, which is crucial in the proof of theorem 2.1.

Proposition 4.1 Assume that $r \in W^{3, \infty}\left(\mathbb{R}^{2}\right)$. Then, for any $\varepsilon$ satisfying (??), we have

$$
\left\|\widehat{u}_{\varepsilon}-\bar{u}\right\|_{H^{1}(\Omega)} \leq C \sqrt{\varepsilon}
$$

and

$$
\left\|\widehat{p}_{\varepsilon}-p_{0}\right\|_{L^{2}(\Omega)} \leq C \sqrt{\varepsilon}
$$

where

$$
\bar{u}=u_{0}+\varepsilon x_{3} \bar{\eta}^{\varepsilon} \partial_{x_{3}} u_{0}
$$

and $C$ is independent of $\varepsilon$.

This result is proved in Section 6. Before, we need some technical results, which are given in Section 5.

Remark 4.1 Proceeding as in [?], it can be seen that the assumption $r \in W^{3, \infty}\left(\mathbb{R}^{2}\right)$ leads to the regularity of $\left(u_{0}, p_{0}\right)$, namely $u_{0} \in\left(H^{3}(\Omega)\right)^{3}$ and $p_{0} \in H^{2}(\Omega)$. This will be used in the sequel. 


\section{Some technical lemmas}

Lemma 5.1 Let $\gamma=\gamma\left(x^{\prime}, y^{\prime}\right)$ be a Lipschitz function on $S^{2}$, periodic with respect to $y^{\prime}$ and satisfying

$$
\int_{S} \gamma\left(x^{\prime}, y^{\prime}\right) d y^{\prime}=0
$$

for any $x^{\prime} \in S$. There exists a real number $C$ such that:

i) For all functions $\varphi$ and $\psi$ in $H^{1}(\Omega)$ and $0<\varepsilon \leq 1$, we have

$$
\left|\int_{\Omega} \gamma\left(x^{\prime}, \frac{x^{\prime}}{\varepsilon}\right) \psi(x) \varphi(x) d x\right| \leq C \varepsilon\|\psi\|_{H^{1}(\Omega)}\|\varphi\|_{H^{1}(\Omega)} .
$$

ii) For all functions $\psi \in H^{2}(\Omega)$ and $\varphi \in H^{1}(\Omega)$ and $0<\varepsilon \leq 1$, we have

$$
\left|\int_{S} \gamma\left(x^{\prime}, \frac{x^{\prime}}{\varepsilon}\right) \varphi\left(x^{\prime}, r\left(x^{\prime}\right)\right) \psi\left(x^{\prime}, r\left(x^{\prime}\right)\right) d x^{\prime}\right| \leq C \sqrt{\varepsilon}\|\psi\|_{H^{2}(\Omega)}\|\varphi\|_{H^{1}(\Omega)} .
$$

For the proof, see O.A. Oleinik, A.S. Shamaev and G.A. Yosifian [?, Lemma 1.6, p. 8].

Lemma 5.2 Assume $r \in W^{3, \infty}(S)$. There exists a positive number $C$, independent of $u_{0}$ and $\varepsilon$, with the following properties :

i) For $0<\varepsilon \leq 1$, we have

$$
\left\|\nabla \cdot\left({ }^{t} N_{\varepsilon} \bar{u}\right)\right\|_{L^{2}(\Omega)} \leq C \varepsilon\left\|u_{0}\right\|_{H^{2}(\Omega)} .
$$

ii) For any function $\varphi \in\left(H^{1}(\Omega)\right)^{3}$ and $0<\varepsilon \leq 1$, we have

$$
\left|\int_{\Omega}\left(\widehat{e}_{\varepsilon}(\bar{u}): \widehat{e}_{\varepsilon}(\varphi)\right) j_{\varepsilon}-\int_{\Omega} e\left(u_{0}\right): e(\varphi)\right| \leq C \sqrt{\varepsilon}\left\|u_{0}\right\|_{H^{3}(\Omega)}\|\varphi\|_{H^{1}(\Omega)} .
$$

Proof: Notice that $u_{0} \in\left(H^{3}(\Omega)\right)^{3}$, since $r \in W^{3, \infty}(S)$. Let us first prove (??). Since by definition $N_{\varepsilon}=\left(1+\varepsilon \bar{\eta}^{\varepsilon}\right) M_{\varepsilon}$, we see that

$$
\begin{aligned}
\nabla \cdot\left({ }^{t} N_{\varepsilon} w\right)= & \partial_{x_{1}}\left(\left(1+\varepsilon \bar{\eta}^{\varepsilon}\right) w_{1}\right)+\partial_{x_{2}}\left(\left(1+\varepsilon \bar{\eta}^{\varepsilon}\right) w_{2}\right) \\
& +\partial_{x_{3}}\left(-x_{3} \varepsilon\left(\partial_{x_{1}} \bar{\eta}^{\varepsilon}\right) w_{1}-x_{3} \varepsilon\left(\partial_{x_{2}} \bar{\eta}^{\varepsilon}\right) w_{2}+w_{3}\right) \\
= & \nabla \cdot w+\varepsilon \bar{\eta}^{\varepsilon}\left(\partial_{x_{1}} w_{1}+\partial_{x_{2}} w_{2}\right)-x_{3}\left(\varepsilon\left(\partial_{x_{1}} \bar{\eta}^{\varepsilon}\right) \partial_{x_{3}} w_{1}+\varepsilon\left(\partial_{x_{2}} \bar{\eta}^{\varepsilon}\right) \partial_{x_{3}} w_{2}\right)
\end{aligned}
$$


for any function $w$. With the particular choice $w=\bar{u}=u_{0}+x_{3} \varepsilon \bar{\eta}^{\varepsilon} \partial_{x_{3}} u_{0}$, since $\nabla \cdot u_{0}=0$, we obtain

$$
\nabla \cdot \bar{u}=x_{3}\left(\varepsilon\left(\partial_{x_{1}} \bar{\eta}^{\varepsilon}\right) \partial_{x_{3}} u_{01}+\varepsilon\left(\partial_{x_{2}} \bar{\eta}^{\varepsilon}\right) \partial_{x_{3}} u_{02}\right)+\varepsilon \bar{\eta}^{\varepsilon} \partial_{x_{3}} u_{03}
$$

and

$$
\begin{gathered}
\varepsilon \bar{\eta}^{\varepsilon} \partial_{x_{1}} \bar{u}_{1}-x_{3} \varepsilon\left(\partial_{x_{1}} \bar{\eta}^{\varepsilon}\right) \partial_{x_{3}} \bar{u}_{1}=\varepsilon \bar{\eta}^{\varepsilon} \partial_{x_{1}} u_{01}-x_{3} \varepsilon\left(\partial_{x_{1}} \bar{\eta}^{\varepsilon}\right) \partial_{x_{3}} u_{01} \\
+x_{3}\left(\varepsilon \bar{\eta}^{\varepsilon}\right)^{2} \partial_{x_{1} x_{3}}^{2} u_{01}-x_{3}^{2} \varepsilon^{2} \bar{\eta}^{\varepsilon}\left(\partial_{x_{1}} \bar{\eta}^{\varepsilon}\right) \partial_{x_{3} x_{3}}^{2} u_{01}
\end{gathered}
$$

Using the corresponding similar equality for $\bar{u}_{2}$, we deduce that

$$
\begin{aligned}
\nabla \cdot\left({ }^{t} N_{\varepsilon} \bar{u}\right)= & x_{3}\left(\varepsilon \bar{\eta}^{\varepsilon}\right)^{2}\left(\partial_{x_{1} x_{3}}^{2} u_{01}+\partial_{x_{2} x_{3}}^{2} u_{02}\right) \\
& -x_{3}^{2} \varepsilon \bar{\eta}^{\varepsilon}\left(\varepsilon\left(\partial_{x_{1}} \bar{\eta}^{\varepsilon}\right) \partial_{x_{3} x_{3}}^{2} u_{01}+\varepsilon\left(\partial_{x_{2}} \bar{\eta}^{\varepsilon}\right) \partial_{x_{3} x_{3}}^{2} u_{02}\right) .
\end{aligned}
$$

The inequality (??) follows, since $\left|\bar{\eta}^{\varepsilon}\left(x^{\prime}\right)\right| \leq\|\eta\|_{L^{\infty}\left(S^{2}\right)}$ and

$$
\left|\varepsilon \partial_{x_{i}} \bar{\eta}^{\varepsilon}\left(x^{\prime}\right)\right|=\left|\varepsilon \partial_{x_{i}} \eta\left(x^{\prime}, \frac{x^{\prime}}{\varepsilon}\right)+\partial_{y_{i}} \eta\left(x^{\prime}, \frac{x^{\prime}}{\varepsilon}\right)\right| \leq 2\|\eta\|_{W^{1, \infty}\left(S^{2}\right)}
$$

Let us now prove (??). The definition (??) of $\widehat{e}_{\varepsilon}$ yields the following for all $\bar{u}$ and $\varphi$ :

$$
\left(\widehat{e}_{\varepsilon}(\bar{u}): \widehat{e}_{\varepsilon}(\varphi)\right)=\frac{1}{2} M_{\varepsilon} \nabla \bar{u}: M_{\varepsilon} \nabla \varphi+\frac{1}{2} M_{\varepsilon} \nabla \bar{u}:{ }^{t}\left(M_{\varepsilon} \nabla \varphi\right) .
$$

Moreover, the definition of $M_{\varepsilon}$ leads to the identities, for $1 \leq k \leq 3$,

$$
\left(M_{\varepsilon} \nabla \varphi\right)_{i k}= \begin{cases}\partial_{x_{i}} \varphi_{k}-\frac{1}{j_{\varepsilon}} x_{3} \varepsilon\left(\partial_{x_{i}} \bar{\eta}^{\varepsilon}\right) \partial_{x_{3}} \varphi_{k} & i=1,2 \\ \partial_{x_{3}} \varphi_{k}-\frac{1}{j_{\varepsilon}} \varepsilon \bar{\eta}^{\varepsilon} \partial_{x_{3}} \varphi_{k} & i=3\end{cases}
$$

For $i=3$, we have used $j_{\varepsilon}=1+\varepsilon \bar{\eta}^{\varepsilon}$ and thus $1 / j_{\varepsilon}=1-\varepsilon \bar{\eta}^{\varepsilon} / j_{\varepsilon}$. In particular, we obtain

$$
\left(M_{\varepsilon} \nabla \bar{u}\right)_{i k}= \begin{cases}\partial_{x_{i}} u_{0 k}+x_{3} \varepsilon \bar{\eta}^{\varepsilon} \partial_{x_{i} x_{3}}^{2} u_{0 k}-\frac{1}{j_{\varepsilon}} \varepsilon^{2} \bar{\eta}^{\varepsilon} \partial_{x_{i}} \bar{\eta}^{\varepsilon} \partial_{x_{3} x_{3}}^{2} u_{0 k} & i=1,2 \\ \partial_{x_{3}} u_{0 k}+\frac{1}{j_{\varepsilon}} x_{3} \varepsilon \bar{\eta}^{\varepsilon} \partial_{x_{3} x_{3}}^{2} u_{0 k} & i=3\end{cases}
$$

Therefore,

$$
\widehat{e}_{\varepsilon}(\bar{u}) j_{\varepsilon}: \widehat{e}_{\varepsilon}(\varphi)-e\left(u_{0}\right): e(\varphi)=\sum_{i=1}^{2} \sum_{k=1}^{3} a_{i k}+\sum_{\alpha} \varepsilon \bar{\eta}^{\varepsilon} b_{\alpha}
$$

where

$$
a_{i k}=-\frac{1}{2} x_{3} \varepsilon\left(\partial_{x_{i}} \bar{\eta}^{\varepsilon}\right) \partial_{x_{3}} \varphi_{k}\left(\partial_{x_{i}} u_{0 k}+\partial_{x_{k}} u_{0 i}\right)
$$


and each $b_{\alpha}$ is the product of a first or second derivative of $u_{0}$, a derivative of $\varphi$ and some of the following functions : $x_{3}, j_{\varepsilon},\left(j_{\varepsilon}\right)^{-1}, \varepsilon \bar{\eta}^{\varepsilon}$ and $\varepsilon \partial_{x_{i}} \bar{\eta}^{\varepsilon}$. Since $\left|\varepsilon \bar{\eta}^{\varepsilon}\right| \leq C \varepsilon$, we see that

$$
\int_{\Omega}\left|\varepsilon \bar{\eta}^{\varepsilon} b_{\alpha}\right| \leq C \varepsilon\left\|u_{0}\right\|_{H^{2}(\Omega)}\|\varphi\|_{H^{1}(\Omega)} .
$$

Since

$$
\varepsilon \partial_{x_{i}} \bar{\eta}^{\varepsilon}\left(x^{\prime}\right)=\varepsilon \partial_{x_{i}} \eta\left(x^{\prime}, \frac{x^{\prime}}{\varepsilon}\right)+\partial_{y_{i}} \eta\left(x^{\prime}, \frac{x^{\prime}}{\varepsilon}\right)
$$

we can also put

$$
a_{i k}=-\frac{1}{2} x_{3}{\overline{\partial_{y_{i}} \eta}}^{\varepsilon} \partial_{x_{3}} \varphi_{k}\left(\partial_{x_{i}} u_{0 k}+\partial_{x_{k}} u_{0 i}\right)+\varepsilon \overline{\partial_{x_{i}} \eta} b_{i k}^{\prime},
$$

where the $b_{i k}^{\prime}$ have the same structure as $b_{\alpha}$. Hence,

$$
\int_{\Omega}\left|\varepsilon{\overline{\partial_{x_{i}} \eta}}^{\varepsilon} b_{i k}^{\prime}\right| \leq C \varepsilon\left\|u_{0}\right\|_{H^{2}(\Omega)}\|\varphi\|_{H^{1}(\Omega)} .
$$

The integrals of the other terms in the right-hand side of (??) are as follows :

$$
\begin{aligned}
\int_{\Omega}- & x_{3}{\overline{\partial_{y_{j}} \eta}}^{\varepsilon}\left(\partial_{x_{3}} \varphi_{k}\right)\left(\partial_{x_{l}} u_{0 m}\right) \\
& =\int_{\Omega} \bar{\partial}_{y_{j}} \eta^{\varepsilon} \partial_{x_{3}}\left(x_{3} \partial_{x_{l}} u_{0 m}\right) \varphi_{k}-\int_{\partial \Omega} x_{3} \overline{\partial_{y_{j}} \eta} \varphi_{k}\left(\partial_{x_{l}} u_{0 m}\right) n_{3} d s .
\end{aligned}
$$

Since $1 / \varepsilon$ is an integer and $\eta$ is periodic with respect to all its variables, we have

$$
\int_{S}{\overline{\partial_{y_{j}}}}^{\varepsilon}=\int_{S} \partial_{y_{i}} \eta\left(x^{\prime}, \frac{x^{\prime}}{\varepsilon}\right) d x^{\prime}=0 .
$$

Therefore, thanks to lemma 5.1, part i),

$$
\left|\int_{\Omega} \overline{\partial_{y_{j}} \eta} \partial_{x_{3}}\left(x_{3} \partial_{x_{l}} u_{0 m}\right) \varphi_{k} d x\right| \leq C \varepsilon\left\|u_{0 m}\right\|_{H^{2}(\Omega)}\left\|\varphi_{k}\right\|_{H^{1}(\Omega)} .
$$

On the other hand, from lemma 5.1, part ii), we have

$$
\begin{gathered}
\left|\int_{\partial \Omega} \overline{\partial_{y_{j}} \eta} x^{\varepsilon} x_{3}\left(\partial_{x_{l}} u_{0 m}\right) \varphi_{k} n_{3} d s\right| \\
\leq\left|\int_{S} \partial_{y_{j}} \eta\left(x^{\prime}, \frac{x^{\prime}}{\varepsilon}\right)\left(x_{3}\left(\partial_{x_{l}} u_{0 m}\right) \varphi_{k}\right)\left(x^{\prime}, r\left(x^{\prime}\right)\right) d x^{\prime}\right| \\
\leq \sqrt{\varepsilon}\left\|u_{0 m}\right\|_{H^{3}\left(\Omega_{\varepsilon}\right)}\left\|\varphi_{k}\right\|_{H^{1}\left(\Omega_{\varepsilon}\right)} .
\end{gathered}
$$

Here, we have used that $n_{3} d s=d x^{\prime}$. This proves that

$$
\left|\int_{\Omega} a_{i, k}\right| \leq C \varepsilon\left\|u_{0}\right\|_{H^{3}(\Omega)}\left\|\varphi_{k}\right\|_{H^{1}(\Omega)} .
$$

This completes the proof of (??). 
Lemma 5.3 Let us assume that $r \in W^{3, \infty}(S)$. Then, for any $\varepsilon$ satisfying (??) and any $\varphi \in\left(H_{\mathrm{per}}^{1}(\Omega)\right)^{3}$, we have

$$
\left|\int_{\Omega} p_{0} \nabla \cdot\left({ }^{t} N_{\varepsilon} \varphi-\varphi\right)\right| \leq C \sqrt{\varepsilon}\left\|p_{0}\right\|_{H^{2}(\Omega)}\|\varphi\|_{H^{1}(\Omega)},
$$

where $C$ is independent of $\varepsilon, p_{0}$ and $\varphi$.

Proof: Let us first notice that $p_{0} \in H^{2}(\Omega)$, since $r \in W^{3, \infty}(S)$. We have

$$
\nabla \cdot\left({ }^{t} N_{\varepsilon} \varphi-\varphi\right)=\varepsilon \bar{\eta}^{\varepsilon}\left(\partial_{x_{1}} \varphi_{1}+\partial_{x_{2}} \varphi_{2}\right)-x_{3} \sum_{j=1}^{2}\left(\varepsilon{\overline{\partial_{x_{j}}}}^{\varepsilon}+{\overline{\partial_{y_{j}} \eta}}^{\varepsilon}\right) \partial_{x_{3}} \varphi_{j}
$$

Then, multiplying by $p_{0}$ and integrating by parts the last term, we see that

$$
\begin{gathered}
\int_{\Omega} p_{0} \nabla \cdot\left({ }^{t} N_{\varepsilon} \varphi-\varphi\right)=\int_{\Omega} \varepsilon p_{0} \sum_{j=1}^{2}\left(\bar{\eta}^{\varepsilon} \partial_{x_{j}} \varphi_{j}-x_{3} \overline{\partial_{x_{j}} \eta} \partial_{x_{3}} \varphi_{j}\right) \\
+\int_{\Omega} \partial_{x_{3}}\left(x_{3} p_{0}\right) \sum_{j=1}^{2}{\overline{\partial_{y_{j}}}}^{\varepsilon} \varphi_{j}-\int_{S} r\left(x^{\prime}\right) p_{0}\left(x^{\prime}, r\left(x^{\prime}\right)\right) \sum_{j=1}^{2} \overline{\partial_{y_{j}} \eta} \bar{\eta}^{\varepsilon}\left(x^{\prime}\right) \varphi_{j}\left(x^{\prime}, r\left(x^{\prime}\right)\right) d x^{\prime} .
\end{gathered}
$$

In the right-hand side, the first integral is bounded by

$$
\left|\int_{\Omega} \varepsilon p_{0} \sum_{j=1}^{2}\left(\bar{\eta}^{\varepsilon} \partial_{j} \varphi_{j}-\varepsilon x_{3} \overline{\partial_{x_{j}} \eta} \partial_{x_{3}} \varphi_{j}\right)\right| \leq C \varepsilon\left\|p_{0}\right\|_{L^{2}}\left\|\varphi_{j}\right\|_{H^{1}(\Omega)}
$$

Using lemma 5.1, part i) and (??), the second integral is bounded by

$$
\left|\int_{\Omega} \partial_{x_{3}}\left(x_{3} p_{0}\right) \sum_{j=1}^{2} \overline{\partial_{y_{j}} \eta} \varphi_{j}\right| \leq C \varepsilon\left\|p_{0}\right\|_{H^{2}(\Omega)}\left\|\varphi_{j}\right\|_{H^{1}(\Omega)}
$$

On the other hand, from lemma 5.1, part ii), the boundary integral satisfies

$$
\left|\int_{S} r\left(x^{\prime}\right) p_{0}\left(x^{\prime}, r\left(x^{\prime}\right)\right) \sum_{j=1}^{2}{\overline{\partial_{y_{j}}}}^{\varepsilon}\left(x^{\prime}\right) \varphi_{j}\left(x^{\prime}, r\left(x^{\prime}\right)\right) d x^{\prime}\right| \leq C \sqrt{\varepsilon}\left\|p_{0}\right\|_{H^{2}(\Omega)}\|\varphi\|_{H^{1}(\Omega)} .
$$

Hence, lemma 5.3 is proved.

Lemma 5.4 Assume that $\psi \in L_{\mathrm{per}}^{2}(\Omega)$ is such that $\int_{\Omega} \psi=0$. Then there exists $\varphi \in\left(H_{0}^{1}(\Omega)\right)^{3}$ such that

$$
\nabla \cdot{ }^{t}\left(N_{\varepsilon} \varphi\right)=\psi \text { in } \Omega, \quad\|\varphi\|_{H^{1}(\Omega)} \leq C\|\psi\|_{L^{2}(\Omega)},
$$

where $C$ is independent of $\varepsilon, \psi$ and $\varphi$. 
ProOF: Let us put

$$
\psi_{\varepsilon}=\frac{1}{1+\varepsilon \bar{\eta}^{\varepsilon}}\left(\psi \circ \mathcal{L}_{\varepsilon}^{-1}\right) .
$$

Clearly, $\psi_{\varepsilon} \in L_{\text {per }}^{2}\left(\Omega_{\varepsilon}\right)$. From lemma 3.4 with $w_{0}=0$, there exists $w_{\varepsilon} \in\left(H_{0}^{1}\left(\Omega_{\varepsilon}\right)\right)^{3}$ such that $\nabla \cdot w_{\varepsilon}=\psi_{\varepsilon}$ in $\Omega_{\varepsilon}$ and

$$
\left\|w_{\varepsilon}\right\|_{H^{1}\left(\Omega_{\varepsilon}\right)} \leq C\left\|\psi_{\varepsilon}\right\|_{L^{2}\left(\Omega_{\varepsilon}\right)},
$$

where $C$ does not depend of $\varepsilon, \psi_{\varepsilon}$ and $w_{\varepsilon}$. In accordance with (??), the function $\varphi=\widehat{w}_{\varepsilon}$ satisfies $\nabla \cdot\left({ }^{t} N_{\varepsilon} \varphi\right)=\psi$ in $\Omega$. Moreover,

$$
\int_{\Omega_{\varepsilon}}\left|\psi_{\varepsilon}\right|^{2}=\int_{\Omega} \frac{1}{1+\varepsilon \bar{\eta}^{\varepsilon}}|\psi|^{2} \leq \int_{\Omega}|\psi|^{2}
$$

\section{The proofs of proposition 4.1 and theorem 2.1}

Proof of PROposition 4.1: We will first proof the estimates (??). Let us write the weak formulation of the problem satisfied by $\left(u_{0}, p_{0}\right)$ :

$$
\begin{gathered}
\left(u_{0}, p_{0}\right) \in\left(H_{\mathrm{per}}^{1}(\Omega)\right)^{3} \times L_{\mathrm{per}}^{2}(\Omega), \\
2 \nu \int_{\Omega} e\left(u_{0}\right): e(\varphi)-\int_{\Omega} p_{0} \nabla \cdot \varphi+\int_{R} K u_{\varepsilon} \cdot \varphi+k \int_{P} u_{\varepsilon} \cdot \varphi=k \int_{P} g \cdot \varphi \\
\forall \varphi \in\left(H_{\mathrm{per}}^{1}(\Omega)\right)^{3}, \\
\nabla \cdot u_{0}=0 \text { in } \Omega .
\end{gathered}
$$

Substracting (??) from (??), we obtain, for all $\varphi \in\left(H_{\text {per }}^{1}(\Omega)\right)^{3}$,

$$
\begin{gathered}
2 \nu \int_{\Omega}\left(\widehat{e}_{\varepsilon}\left(\widehat{u}_{\varepsilon}\right): \widehat{e}_{\varepsilon}(\varphi) j_{\varepsilon}-e\left(u_{0}\right): e(\varphi)\right)-\int_{\Omega}\left(\widehat{p}_{\varepsilon} \nabla \cdot\left({ }^{t} N_{\varepsilon} \varphi\right)-p_{0} \nabla \cdot \varphi\right) \\
+\int_{R}\left(k \mu_{\varepsilon} \widehat{u}_{\varepsilon}-K u_{0}\right) \cdot \varphi d s+k \int_{P}\left(\widehat{u}_{\varepsilon}-u_{0}\right) \cdot \varphi d s=0 .
\end{gathered}
$$

Let us set $\widehat{u}_{\varepsilon}=\bar{u}+z_{\varepsilon}$, where $\bar{u}=u_{0}+x_{3} \varepsilon \bar{\eta}^{\varepsilon} \partial_{x_{3}} u_{0}$. Then (??) reads

$$
\left\|z_{\varepsilon}\right\|_{H^{1}(\Omega)} \leq C \sqrt{\varepsilon}
$$

Since $\bar{u}=u_{0}$ on $P,(? ?)$ gives :

$$
\begin{gathered}
2 \nu \int_{\Omega}\left(\widehat{e}_{\varepsilon}\left(z_{\varepsilon}\right): \widehat{e}_{\varepsilon}(\varphi)\right) j_{\varepsilon}+k \int_{R} z_{\varepsilon} \cdot \varphi \mu_{\varepsilon} d s+k \int_{P} z_{\varepsilon} \cdot \varphi d s \\
=-2 \nu \int_{\Omega}\left(\widehat{e}_{\varepsilon}(\bar{u}): \widehat{e}_{\varepsilon}(\varphi) j_{\varepsilon}-e\left(u_{0}\right): e(\varphi)\right)+\int_{\Omega}\left(\widehat{p}_{\varepsilon}-p_{0}\right) \nabla \cdot\left({ }^{t} N_{\varepsilon} \varphi\right) \\
+\int_{\Omega} p_{0} \nabla \cdot\left({ }^{t} N_{\varepsilon} \varphi-\varphi\right)-\int_{R}\left(k \mu_{\varepsilon} \bar{u}-K u_{0}\right) \cdot \varphi d s .
\end{gathered}
$$


Let us choose $\varphi=z_{\varepsilon}$. Then the left-hand side is larger than $C\left\|z_{\varepsilon}\right\|_{\left(H^{1}(\Omega)\right)^{3}}^{2}$ thanks to Korn inequality (??). Using (??) to estimate the first integral in the right-hand side of (??), we see that

$$
\left\|z_{\varepsilon}\right\|_{H^{1}(\Omega)}^{2} \leq C \sqrt{\varepsilon}\left\|u_{0}\right\|_{H^{3}(\Omega)}\left\|z_{\varepsilon}\right\|_{H^{1}(\Omega)}+A+B+\left|\int_{R}\left(k \mu_{\varepsilon} \bar{u}-K u_{0}\right) \cdot z_{\varepsilon} d s\right|,
$$

where $C$ is independent of $\varepsilon$ and $A$ and $B$ are respectively given by

$$
A=\left|\int_{\Omega}\left(\widehat{p}_{\varepsilon}-p_{0}\right) \nabla \cdot\left({ }^{t} N_{\varepsilon} z_{\varepsilon}\right)\right|, \quad B=\left|\int_{\Omega} p_{0} \nabla \cdot\left({ }^{t} N_{\varepsilon} z_{\varepsilon}-z_{\varepsilon}\right)\right| .
$$

Thanks to (??),

$$
\nabla \cdot\left({ }^{t} N_{\varepsilon} z_{\varepsilon}\right)=\nabla \cdot\left({ }^{t} N_{\varepsilon} \widehat{u}_{\varepsilon}\right)-\nabla \cdot\left({ }^{t} N_{\varepsilon} \bar{u}\right)=-\nabla \cdot\left({ }^{t} N_{\varepsilon} \bar{u}\right) .
$$

Thus, (??) and (??) give

$$
A \leq C \varepsilon\left(\left\|\widehat{p}_{\varepsilon}\right\|_{L^{2}(\Omega)}+\left\|p_{0}\right\|_{L^{2}(\Omega)}\right)\left\|u_{0}\right\|_{H^{2}(\Omega)} \leq C \varepsilon .
$$

On the other hand, (??) implies

$$
B \leq C \sqrt{\varepsilon}\left\|p_{0}\right\|_{H^{2}(\Omega)}\left\|z_{\varepsilon}\right\|_{H^{1}(\Omega)} \leq C \sqrt{\varepsilon}\left\|z_{\varepsilon}\right\|_{H^{1}(\Omega)} .
$$

In order to estimate the integral over $R$ in (??), let us put

$$
k \mu_{\varepsilon} \bar{u}-K u_{0}=\left(k \bar{m}^{\varepsilon}-K\right) u_{0}+k\left(\mu_{\varepsilon}-\bar{m}^{\varepsilon}\right) u_{0}+k \mu_{\varepsilon}\left(\bar{u}-u_{0}\right),
$$

where $\bar{m}^{\varepsilon}\left(x^{\prime}\right)=m\left(x^{\prime}, x^{\prime} / \varepsilon\right)$. From the definition of $K$, we see that $\left(k \bar{m}^{\varepsilon}-K\right)\left(x^{\prime}\right)=$ $k \gamma\left(x^{\prime}, x^{\prime} / \varepsilon\right)$, where

$$
\gamma\left(x^{\prime}, y^{\prime}\right)=m\left(x^{\prime}, y^{\prime}\right)-\langle m\rangle\left(x^{\prime}\right) .
$$

Thus, lemma 5.1, part ii), gives :

$$
\left|\int_{R}\left(k \bar{m}^{\varepsilon}-K\right) u_{0} \cdot z_{\varepsilon} d s\right| \leq C \sqrt{\varepsilon}\left\|u_{0}\right\|_{H^{2}(\Omega)}\left\|z_{\varepsilon}\right\|_{H^{1}(\Omega)} .
$$

From (??), (??) and (??), we deduce that

$$
\mu_{\varepsilon}^{2}-\left(\bar{m}^{\varepsilon}\right)^{2}=\frac{\left|\varepsilon r{\overline{\nabla_{x^{\prime}} \eta}}^{\varepsilon}+\varepsilon \bar{\eta}^{\varepsilon} \nabla r\right|^{2}+2\left(\varepsilon r{\overline{\nabla_{x^{\prime}} \eta}}^{\varepsilon}+\varepsilon \bar{\eta}^{\varepsilon} \nabla r\right)\left(r{\overline{\nabla_{y^{\prime}} \eta}}^{\varepsilon}+\nabla r\right)}{1+|\nabla r|^{2}} .
$$

This implies that $\left|\mu_{\varepsilon}-\bar{m}^{\varepsilon}\right| \leq C \varepsilon$ and

$$
\left|\int_{R} k\left(\mu_{\varepsilon}-\bar{m}^{\varepsilon}\right) u_{0} \cdot z_{\varepsilon} d s\right| \leq C \varepsilon\left\|u_{0}\right\|_{H^{1}(\Omega)}\left\|z_{\varepsilon}\right\|_{H^{1}(\Omega)} .
$$


Finally, $\left|\bar{u}-u_{0}\right|=\left|x_{3} \bar{\eta}^{\varepsilon} \partial_{x_{3}} u_{0}\right| \leq C\left|\partial_{x_{3}} u_{0}\right|$ and

$$
\left|\int_{R} k \mu_{\varepsilon}\left(\bar{u}-u_{0}\right) \cdot z_{\varepsilon} d s\right| \leq C \varepsilon\left\|u_{0}\right\|_{H^{2}(\Omega)}\left\|z_{\varepsilon}\right\|_{H^{1}(\Omega)}
$$

From (??), (??), (??) and (??), we see that

$$
\left|\int_{R}\left(k \mu_{\varepsilon} \bar{u}-K u_{0}\right) \cdot z_{\varepsilon} d s\right| \leq C \sqrt{\varepsilon}\left\|z_{\varepsilon}\right\|_{H^{1}(\Omega)} .
$$

Using (??), (??) and (??) in (??), we obtain

$$
\left\|z_{\varepsilon}\right\|_{H^{1}(\Omega)}^{2} \leq C \sqrt{\varepsilon}\left\|z_{\varepsilon}\right\|_{H^{1}(\Omega)}+C \varepsilon .
$$

This implies (??) and, therefore, (??) holds.

Let us now prove (??). Choosing $\varphi \in\left(H_{0}^{1}(\Omega)\right)^{3}$ in (??), we find

$$
\begin{gathered}
2 \nu \int_{\Omega}\left(\widehat{e}_{\varepsilon}\left(z_{\varepsilon}\right): \widehat{e}_{\varepsilon}(\varphi)\right) j_{\varepsilon}=\int_{\Omega}\left(\widehat{p}_{\varepsilon}-p_{0}\right) \nabla \cdot\left({ }^{t} N_{\varepsilon} \varphi\right)+\int_{\Omega} p_{0} \nabla \cdot\left({ }^{t} N_{\varepsilon} \varphi-\varphi\right) \\
-2 \nu \int_{\Omega}\left(\left(\widehat{e}_{\varepsilon}(\bar{u}): \widehat{e}_{\varepsilon}(\varphi)\right) j_{\varepsilon}-e\left(u_{0}\right): e(\varphi)\right) .
\end{gathered}
$$

Let $\psi$ be a function in $L_{\mathrm{per}}^{2}(\Omega)$ and let $\varphi \in\left(H_{0}^{1}(\Omega)\right)^{3}$ be the function furnished by lemma 5.4. Then (??) reads

$$
\begin{gathered}
\int_{\Omega}\left(\widehat{p}_{\varepsilon}-p_{0}\right) \psi=2 \nu \int_{\Omega}\left(\widehat{e}_{\varepsilon}\left(z_{\varepsilon}\right): \widehat{e}_{\varepsilon}(\varphi)\right) j_{\varepsilon} \\
+2 \nu \int_{\Omega}\left(\left(\widehat{e}_{\varepsilon}(\bar{u}): \widehat{e}_{\varepsilon}(\varphi)\right) j_{\varepsilon}-e\left(u_{0}\right): e(\varphi)\right)-\int_{\Omega} p_{0} \nabla \cdot\left({ }^{t} N_{\varepsilon} \varphi-\varphi\right) .
\end{gathered}
$$

Using (??), (??), (??) and (??), it is not hard to see that

$$
\left|\int_{\Omega}\left(\widehat{p}_{\varepsilon}-p_{0}\right) \psi\right| \leq C \sqrt{\varepsilon}\|\psi\|_{L^{2}}
$$

Since $\psi$ is arbitrary, this implies (??). This completes the proof.

Proof of Theorem 2.1: First, let us prove that (??) follows from (??). Let $\delta^{*}=1 /\left(2\|\eta\|_{L^{\infty}\left(S^{2}\right)}\right)$. Thanks to (??), we have

$$
\left|T_{\varepsilon}-T_{0}\right|=\left|k g \cdot \int_{P}\left(u_{0}-u_{\varepsilon}\right)\right| \leq k|g| C_{\delta^{*}}\left\|u_{0}-u_{\varepsilon}\right\|_{H^{1}\left(\omega_{\delta^{*}}\right)} .
$$

Therefore, (??) implies (??).

Let us now prove (??). It suffices to assume $\delta \leq \delta^{*}$, since (??) for $\delta=\delta^{*}$ implies (??) for any $\delta \geq \delta^{*}$. Using (??) and putting $\widehat{\omega}_{\delta}=\mathcal{L}_{\varepsilon}^{-1}\left(\omega_{\delta}\right)$, we have

$$
\|\varphi\|_{H^{1}\left(\omega_{\delta}\right)}^{2}=\int_{\widehat{\omega}_{\delta}} j_{\varepsilon}\left(|\widehat{\varphi}|^{2}+\left|M_{\varepsilon} \nabla \hat{\varphi}\right|^{2}\right) \leq C\|\widehat{\varphi}\|_{H^{1}\left(\widehat{\omega}_{\delta}\right)}
$$


Then, it suffices to prove that

$$
\left\|\widehat{u}_{\varepsilon}-\widehat{u}_{0}\right\|_{H^{1}\left(\widehat{\omega}_{\delta}\right)} \leq C \sqrt{\varepsilon}
$$

(where $C$ can depend of $\delta$ ). From (??), we see that it is sufficient to prove that

$$
\left\|\bar{u}-\widehat{u}_{0}\right\|_{H^{1}\left(\widehat{\omega}_{\delta}\right)} \leq C \sqrt{\varepsilon} .
$$

Since $\bar{u}=u_{0}=\widehat{u}_{0}$ on $P$, we only have to estimate the $L^{2}$ norms of the first derivatives $\partial_{x_{j}}\left(\bar{u}-\widehat{u}_{0}\right)$. In fact, we are going to prove that, for all $x \in \widehat{\omega}_{\delta}$,

$$
\left|\partial_{x_{j}}\left(\bar{u}-u_{0}\right)(x)\right| \leq C \sqrt{\varepsilon}
$$

Recall that $\bar{u}=u_{0}+\varepsilon x_{3} \bar{\eta}^{\varepsilon} \partial_{x_{3}} u_{0}$. Then,

$$
\partial_{x_{j}} \bar{u}= \begin{cases}\partial_{x_{j}} u_{0}+x_{3}\left({\overline{\partial_{y_{j}} \eta}}^{\varepsilon}+\varepsilon{\overline{\partial_{x_{j}}}}^{\varepsilon}\right) \partial_{x_{3}} u_{0}+\varepsilon x_{3} \bar{\eta}^{\varepsilon} \partial_{x_{3} x_{j}}^{2} u_{0} & j=1,2 \\ \partial_{x_{3}} u_{0}+\varepsilon \bar{\eta}^{\varepsilon} \partial_{x_{3}} u_{0}+\varepsilon x_{3} \bar{\eta}^{\varepsilon} \partial_{x_{3} x_{3}}^{2} u_{0} & j=3\end{cases}
$$

Thanks to (??),

$$
\partial_{x_{j}} \widehat{u}_{0}= \begin{cases}\widehat{\partial_{x_{j}}} u_{0}+x_{3}\left(\overline{\partial_{y_{j}} \eta}+\varepsilon \overline{\partial_{x_{j}} \eta}\right) \widehat{\partial_{x_{3}} u_{0}} & j=1,2, \\ \left(1+\varepsilon \bar{\eta}^{\varepsilon}\right) \widehat{\partial_{x_{3}} u_{0}} & j=3 .\end{cases}
$$

Since $\bar{\eta}^{\varepsilon},{\overline{\partial_{x_{j}} \eta}}^{\varepsilon}$ and ${\overline{\partial_{y_{j}} \eta}}^{\varepsilon}$ are bounded, it follows that

$$
\left.\left|\partial_{x_{j}}\left(\bar{u}-\widehat{u}_{0}\right)\right| \leq C \sum_{k=1}^{3}\left|\partial_{x_{k}} u_{0}-\widehat{\partial_{x_{k}}} u_{0}\right|+C \varepsilon\left(\left|\widehat{\partial_{x_{3}} u_{0}}\right|+\left|\partial_{x_{3}} u_{0}\right|+\left|\partial_{x_{3} x_{j}} u_{0}\right|\right)\right) \text {. }
$$

Since $r \in W^{3, \infty}(S)$, we have $u_{0} \in\left(H^{3}(\Omega)\right)^{3}$ and thus Morrey-Sobolev theorem gives $u_{0} \in\left(C^{1,1 / 2}(\bar{\Omega})\right)^{3}$, with a norm in this space bounded by $C\left\|u_{0}\right\|_{H^{3}(\Omega)}$. Therefore, for all $x \in \widehat{\omega}_{\delta}$,

$$
\left|\left(\partial_{x_{k}} u_{0}-\widehat{\partial_{x_{k}}} u_{0}\right)(x)\right| \leq C_{\delta}\left\|u_{0}\right\|_{H^{3}(\Omega)}\left|x-\mathcal{L}_{\varepsilon}(x)\right|^{1 / 2} \leq C_{\delta} \sqrt{\varepsilon}
$$

and (??) is established. Thanks to Poincaré inequality, (??) holds too. This completes the proof of (??).

Finally, let us prove (??). Arguing as before, it suffices to show that

$$
\left\|\widehat{p}_{\varepsilon}-\widehat{p}_{0}\right\|_{L^{2}\left(\widehat{\omega}_{\delta}\right)} \leq C \sqrt{\varepsilon}
$$

From (??), it suffices to prove that

$$
\left\|p_{0}-\widehat{p}_{0}\right\|_{L^{2}\left(\widehat{\omega}_{\delta}\right)} \leq C \sqrt{\varepsilon}
$$


But we already know that $p_{0} \in H^{2}(\Omega)$, whence (again from Morrey-Sobolev embedding) $p_{0} \in C^{0,1 / 2}(\bar{\Omega})$ and its norm in this space is bounded by $C\left\|p_{0}\right\|_{H^{2}(\Omega)}$. This leads to the following inequalities, for all $x \in \widehat{\omega}_{\delta}$,

$$
\left|\left(p_{0}-\widehat{p}_{0}\right)(x)\right| \leq C_{\delta}\left\|p_{0}\right\|_{H^{2}(\Omega)}\left|x-\mathcal{L}_{\varepsilon}(x)\right|^{1 / 2} \leq C_{\delta} \sqrt{\varepsilon} .
$$

This implies (??) and, thus, (??) holds. The proof of theorem 2.1 is now completed.

Acknowledgements: The work of the second and third authors has been partially supported by D.G.E.S.-Spain, Proyecto PB98-1242. The work of the fourth author has been partially supported by IBERDROLA visiting researchers programme.

\section{References}

[1] G. Allaire - Homogenization of the Navier-Sokes equations with a slip boundary condition, Comm. Pure Appl. Math., XLIV, 6, p. 605-642, 1991.

[2] Y. Amirat, J. Simon - Influence de la rugosité en hydrodynamique linéaire, C. R. Acad. Sci. Paris, t. 323, Série I, p. 313-318, 1996.

[3] Y. Amirat, J. Simon - Riblets and drag minimization. In Optimization methods in pde's, S. Cox and I. Lasiecka eds., Contemporary Mathematics, AMS, p. 9-17, 1997.

[4] Y. Amirat, D. Bresch, J. Lemoine, J. Simon - Effect of rugosity on a flow governed by Navier-Stokes equations, Quarterly Appl. Math., (to appear).

[5] J.A. Bello - $L^{r}$ regularity for the Stokes and Navier-Stokes problems, Ann. Mat. Pura. Appl. Vol. 170, p. 187-206, 1996.

[6] D. Cioranescu, P. Donato and H. I. Ene - Homogenization of the Stokes problem with non-homogeneous slip boundary conditions, Math. Methods Appl. Sci., Vol. 19, p. 857-882, 1996.

[7] C. Conca - On the application of the homogenization theory to a class of problems arising in fluid mechanics, J. Math. Pures et Appl., 64, p. 31-75, 1985.

[8] C. ConcA - Numerical results on the homogeneization of Stokes and NavierStokes equations modeling a class of problems from fluid mechanics, Comput. Methods Appl. Mech. Engrg., 53(3), p. 223-258, 1985. 
[9] G.A. Chechin, A. Friedman, A.L. Pianitski - The boundary-value problem in domains with very rapidly oscillating boundary, J. Math. Anal. Appl., 231, p. 213-234, 1999.

[10] G. Duvaut, J.L. Lions - Les inéquations en Mécanique et Physique, Dunod, Paris 1972.

[11] G.P. GALDI - An introduction to the mathematical theory of the Navier-Stokes equations I : Linearized steady problems, Springer-Verlag, New York 1994.

[12] V. Girault, P.A. RaviarT - Finite element methods for Navier-Stokes Equations, Springer-Verlag, Berlin 1986.

[13] W. JÄGER, A. Mikelić - On the roughness-induced boundary conditions for an incompressible viscous flow, J. Differential Equations (to appear).

[14] R. L. Panton - Incompressible flow, Wiley-Interscience, New-York, 1984.

[15] O.A. Oleinik, A.S. Shamaev, G.A. Yosifian - Mathematical problems in elasticity and homogenization, North-Holland, Amsterdam 1992.

[16] E. SANChez-PALEnCIA - Non-homogeneous media and vibration theory, Lectures Notes in Physics no. 127, Spriger-Verlag, Berlin 1980. 